\title{
A Fronto-Posterior Network Involved in Visual Dimension Changes
}

\author{
S. Pollmann \\ Max-Planck-Institute of Cognitive Neuroscience
}

\section{R. Weidner and H.J. Müller}

University of Leipzig

\section{D.Y. von Cramon}

Max-Planck-Institute of Cognitive Neuroscience, Leipzig

\begin{abstract}
Objects characterized by a unique visual feature may pop out of their environment. When participants have to search for such "odd-one-out" targets, detection is facilitated when targets are consistently defined within the same feature dimension (e.g., color) compared with when the target dimension is uncertain (e.g., color or motion). Further, with dimensional uncertainty, there is a cost when a given target is defined in a different dimension to the preceding target, relative to when the critical dimension remains the same. Behavioral evidence suggests that a target dimension change
\end{abstract}

\section{INTRODUCTION}

Target objects that differ in a single salient attribute from other, distractor, objects in a display can be detected rapidly, irrespective of the number of items in the display. A typical example is a red object among green objects. Subjectively, the odd-one-out item (the target) appears to pop out of the display, and the function relating the detection reaction times (RTs) to the number of display items has a slope close to zero. This popout phenomenon, together with the flat search RT functions, has been taken as support for the idea that certain visual attributes (i.e., features) are registered and compared in parallel across the entire visual field (e.g., Treisman \& Gelade, 1980).

In the typical feature search experiment, both the target dimension (e.g., color) and the target feature value in this dimension (e.g., red) are constant, that is, known to the participant. This contrasts with odd-oneout or singleton feature search in which the target identity is variable across trials and, thus, uncertain. Treisman (1988) examined the effects of two types of uncertainty on search efficiency: In within-dimension search, the target dimension was always constant (e.g., involves a shift of attention to the new dimension. The present fMRI study revealed increased activation in the left frontopolar cortex, as well as in posterior visual areas of the dorsal and ventral streams, specific to changes in the target dimension. In contrast, activation in the striate cortex was decreased. This pattern suggests control of cross-dimensional attention shifts by the frontopolar cortex, modulating visual cortical processing by increased activation in higher-tier visual areas and suppression of activation in lower-tier areas. color), but the target feature value was unpredictable on a trial (blue, green, or red). In cross-dimension search, the target dimension was unpredictable (color, orientation, or size), while the target feature value within a given dimension was constant (e.g., blue). While the slopes of the resulting search RT functions were flat for both types of search, cross-dimension search incurred a constant time cost relative to within-dimension search. Müller, Heller, and Ziegler (1995) replicated this crossdimension search cost relative to the within-dimension condition, in the absence of a within-dimension search cost relative to a new control condition in which both the target dimension and the target feature value were fixed. Müller et al. reasoned that target detection is based on dimension-specific feature contrast or saliency mechanisms (e.g., Wolfe, 1994), which signal the presence of a target within a given dimension, but do not specify its feature value. Subsequently, Found and Müller (1996) described a dimension-specific inter-trial effect: Detection of a target (e.g., a red bar) on a given trial was delayed when the target dimension changed from the preceding to the current trial (e.g., from orientation to color), but not when the target feature 
value changed (e.g., from blue to red). To explain this pattern of results, Müller et al. proposed a "dimensionweighting" account, according to which there is a limit to the total amount of attention, or attentional weight (cf. Duncan \& Humphreys, 1989), available to be allocated to objects' dimensions. Target detection requires that the target dimension be weighted sufficiently to amplify the saliency signal generated within this dimension above the detection threshold. Dimension change incurs a cost because attentional weight must be shifted from the old to the new dimension.

The control task RTs of Müller et al. (1995) revealed no difference between the baseline tasks performed separately (target dimension and feature fixed), suggesting that the tasks were equally difficult. This argues against the cross-dimension search cost (that arises when baseline trials are presented randomized within a single experiment) being due to differential control task difficulty. Instead, it reflects a difficulty to simultaneously attend to multiple dimensions. To explain this difficulty, Treisman proposed that cross-dimension search was parallel within a given dimension, but serial across dimensions (see also the "spatial-and-objectsearch" model of Grossberg, Mingolla, \& Ross, 1994). The assumption of serial cross-dimension search was challenged by Krummenacher, Müller, and Heller (submitted), who showed that search operates in parallel in multiple dimensions, albeit with reduced efficiency compared to search within a single dimension. In terms of the dimension-weighting account, the attentional weight is divided (though not necessarily equally) between multiple dimensions.

One important question concerns whether dimensional weighting is stimulus-driven or expectancy-driven. Müller et al. (1995; Experiment 3) demonstrated a dimension-specific cueing effect, such that precueing a (likely) target dimension expedited detection of targets defined within the likely dimension, whereas precueing a target feature within a fixed dimension had comparatively little effect. However, since the cues were constant throughout a block of trials, the dimension-specific cueing effect could be due to the frequent repetition of targets within the likely dimension (rather than to participants developing and maintaining a dimensionspecific set or expectancy). Work in progress (Reimann, Müller, \& Krummenacher, in preparation) using symbolic trial-by-trial precuing of the likely target dimension suggests that, although the dimension-specific inter-trial effects are largely stimulus-driven, they can be modified by dimensional expectancies. However, in standard cross-dimension search, with the target dimension (and feature) varying unpredictably, participants do not form an explicit memory of the dimensional (or featural) target identity on a given trial, which might carry over as an "expectancy" into the next trial (Müller, Krummenacher, \& Heller, submitted; Experiment 1). Even when made to form such a memory, the pattern of inter-trial effects remains unchanged (Müller et al., submitted; Experiment 2). Taken together, these results indicate that deliberate, expectancy-driven processes do not play a crucial role in dimensional weighting.

The goal of the present fMRI study was to investigate the functional neuroanatomy underlying attentional weighting of, and shifts between, visual dimensions. The dimensions examined were color and motion. Search displays, illustrated in Figure 1, consisted of green square distractors moving sinusoidally along the horizontal axis; targets were defined either by a different movement direction or by a different color. Within blocks, targets were defined by a fixed dimension and feature (control condition), a fixed dimension but a variable feature (within-dimension condition), or a variable dimension (cross-dimension condition). In crossdimension search, shifts between dimensions were hypothesized to activate control structures outside of visual cortex, which exert a top-down influence on

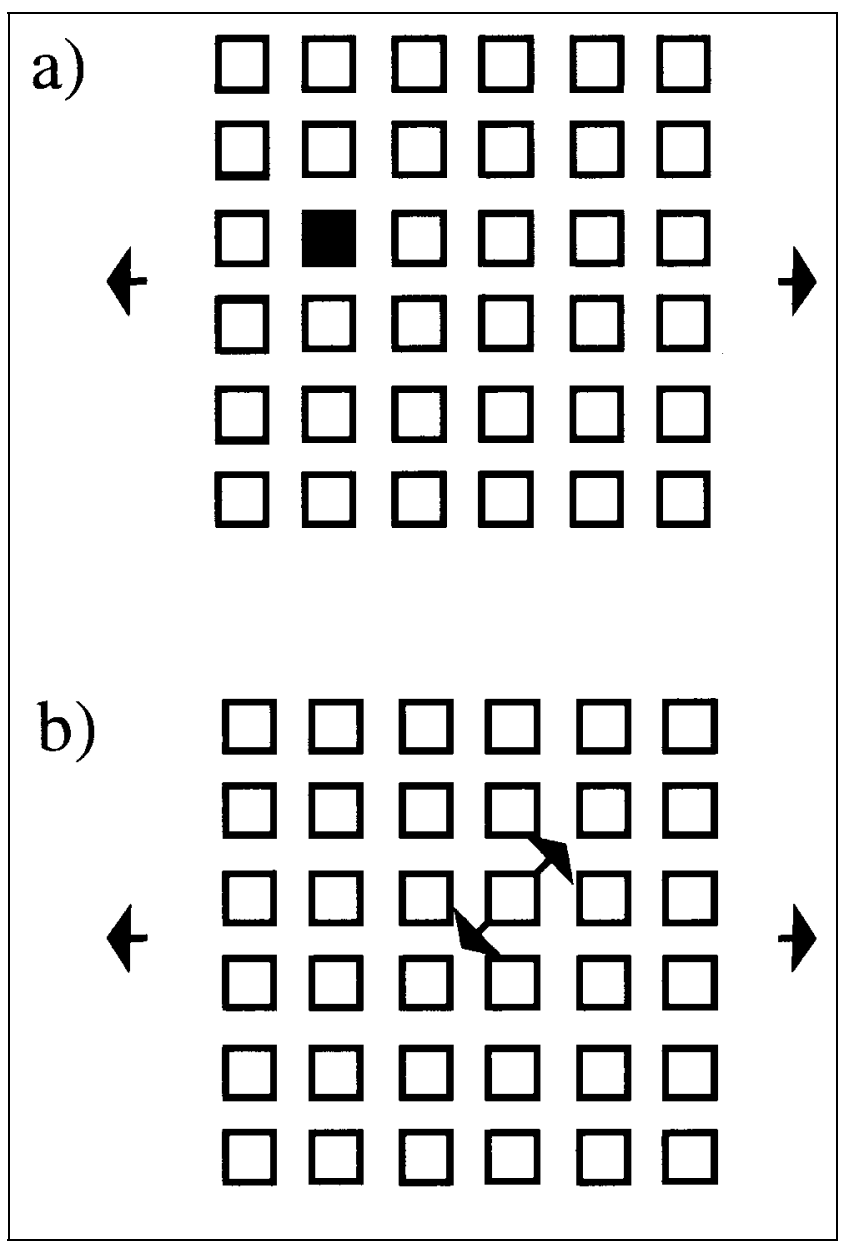

Figure 1. Illustration of stimulus displays in the fMRI study. (a) colordefined target. Open squares indicate green distractors, the filled square indicates a red or blue target, depending on conditions (cf. Methods), arrows indicate direction of sinusoidal movement of the whole display. (b) Motion-defined target. The target square, moving sinusoidally from lower left to upper right (or from upper left to lower right) is indicated by arrows. 
visual areas subserving feature contrast detection within the target dimension (Found \& Müller, 1996) (as used here, the term "top-down" is meant to refer to the anatomical relationship of the brain structures involved, rather than the concept of voluntary, as opposed to automatic, control). The top-down modulation might either increase activation in visual areas, to enhance processing within the new dimension, or decrease activation, to suppress processing within the old dimension. Increases in activation were expected to be manifest in higher-tier visual areas. In contrast, decreases in activation accompanying suppression effects were expected to be more likely in lower-tier visual areas (V1-V3). These predictions derive from Posner and Dehaene's (1994) argument that passive viewing alone leads to near-maximum activation in lower-tier areas, especially V1, leaving little room for further increases in activation due to attention. By the same reasoning, attentive processing is expected to generate greater increases in activation in higher-tier areas, which are less activated under passiveviewing conditions. In support of this, although attention-related increases of the BOLD-response have been reported in V1, they are generally small (Gandhi, Heeger, \& Boynton, 1999; Jäncke, Mirzazade, \& Shah, 1999; Somers, Dale, Seiffert, \& Tootell, 1999; Watanabe et al., 1998). Furthermore, attention to motion has been shown to produce weak blood oxygenation level dependent (BOLD)-response increases in V1, more marked increases in $\mathrm{V} 5$, and strong increases in posterior parietal cortex (Büchel et al., 1998). Finally, spatial attention to a cued display quadrant has been shown to lead to increasing BOLD-response enhancements from V1 to V4 in the representation areas of the attended quadrants; in contrast, the representation areas of the unattended quadrants displayed BOLDresponse reductions that were equal in magnitude in all retinotopic visual areas (Tootell et al., 1998). This pattern of effects is in accord with the hypothesis of Posner and Dehaene (1994).

\section{RESULTS}

\section{Behavioral Data}

The RT data are illustrated in Figure 2a, which presents the mean correct RTs as a function of target (present, absent) and search condition (control, within-dimension, cross-dimension). As can be seen from the figure, search was slowed in the cross-dimension condition relative to both the control and within-dimension search conditions. This cross-dimension search cost was evident with both target-present and target-absent RTs (cost of 31 and $19 \mathrm{msec}$, respectively, relative to within-dimension condition). In contrast, there was no significant cost for within-dimension search relative to the control condition ( 7 and $6 \mathrm{msec}$, respectively). A twoway repeated-measures analysis of variance (ANOVA) revealed the main effects of search condition, $F(2,18)$

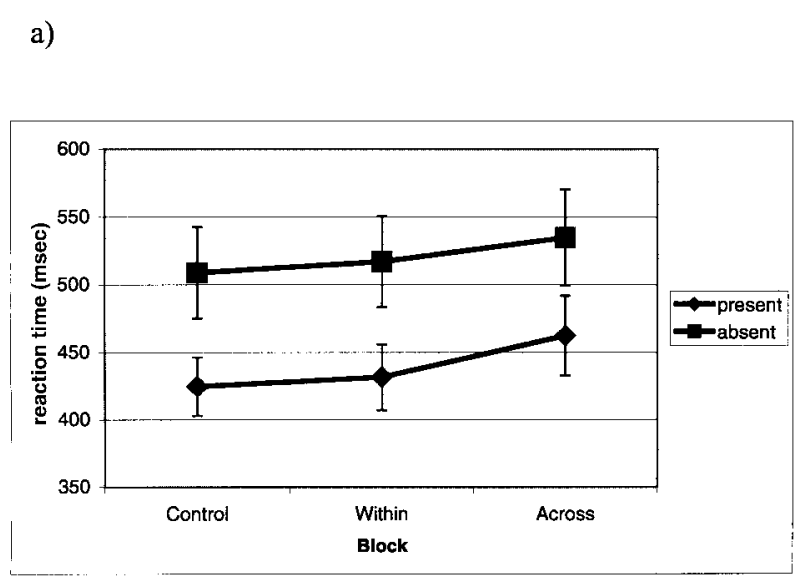

b)

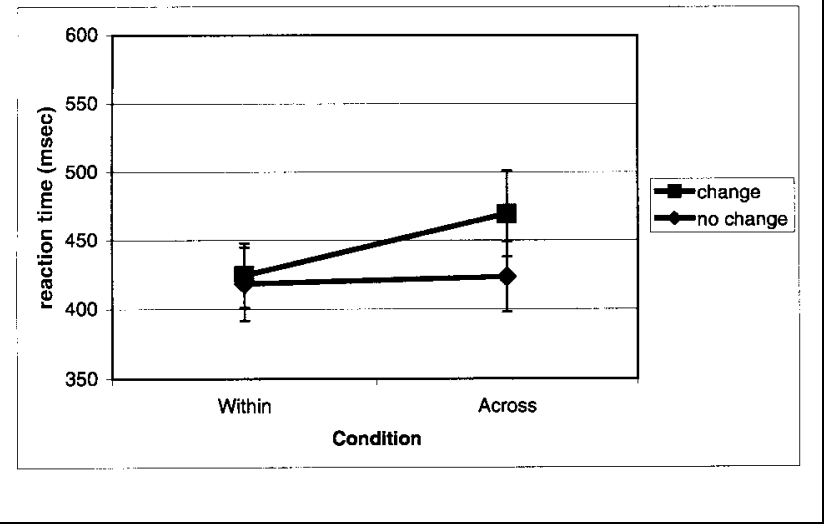

Figure 2. Behavioral data obtained in the fMRI experiment. (a) Reaction times for target-present and absent trials in the control, within-dimension, and cross-dimension search conditions. (b) Reaction times on trial $n$ dependent on the target on trial $n-1$ (change versus no-change), separately for the within-dimension (feature change versus no-change) and the cross-dimension (dimension change versus nochange) search conditions. Bars indicate standard deviations.

$=19.01, M S_{e}=303.30, p<.001$, and target, $F(1,9)=$ $41.09, M S_{e}=2579.29, p<.001$, to be significant. The RT intertrial effects, that is, the RT to a given target on trial $n$ dependent on the identity of the target on the preceding trial $n-1$, are illustrated in Figure $2 \mathrm{~b}$, separately for the within-dimension and cross-dimension conditions. The figure shows that there was a cost associated with a change of target across consecutive trials only when the target-defining dimension changed (i.e., in the crossdimension condition: $48 \mathrm{msec}$ cost), but not when the target-defining feature changed (i.e., in the within-dimension condition: $5 \mathrm{msec}$ cost). A two-way ANOVA of the inter-trial effects, with main terms for search condition (within-dimension, cross-dimension) and change condition (change, no-change), revealed the two main effects and, most importantly, the interaction, $F(1,9)=$ 24.23, $M S_{e}=199.95, p<.001$, to be significant. Planned 
$t$ tests confirmed that there was a significant change cost only in the cross-dimension condition $\left(t_{9}=6.06, p<\right.$ $.001)$, not in the within-dimension condition $\left(t_{9}=.69\right.$, $n s)$. Analogous analyses were performed for the error responses. The error rates were low overall (4.8\%) and reinforced the RT effects. In particular, a change in the target dimension led to a significantly increased rate of target miss errors (5.7\% vs. $2.3 \%$ ), but not a change in the target feature (3.1\% vs. 2.5\%; search condition $\times$ change condition interaction, $F(1,9)=13.49, M S_{e}=$ $.109, p<.005)$. Thus, the pattern of cross-dimension search RT costs together with RT inter-trial effects specific to change of the target-defining dimension neatly replicates the findings of Found and Müller
Figure 3. BOLD-response increases related to the color and movement control conditions versus fixation, the dimensional uncertainty condition versus fixed color and movement dimension blocks, and event-related changes following changes of the target-defining dimension in the dimensional uncertainty condition. The scale indicates activation strengths from $z=4.74$ through $z=12$. The $x$ (-left, + right) coordinate is indicated next to each plane.

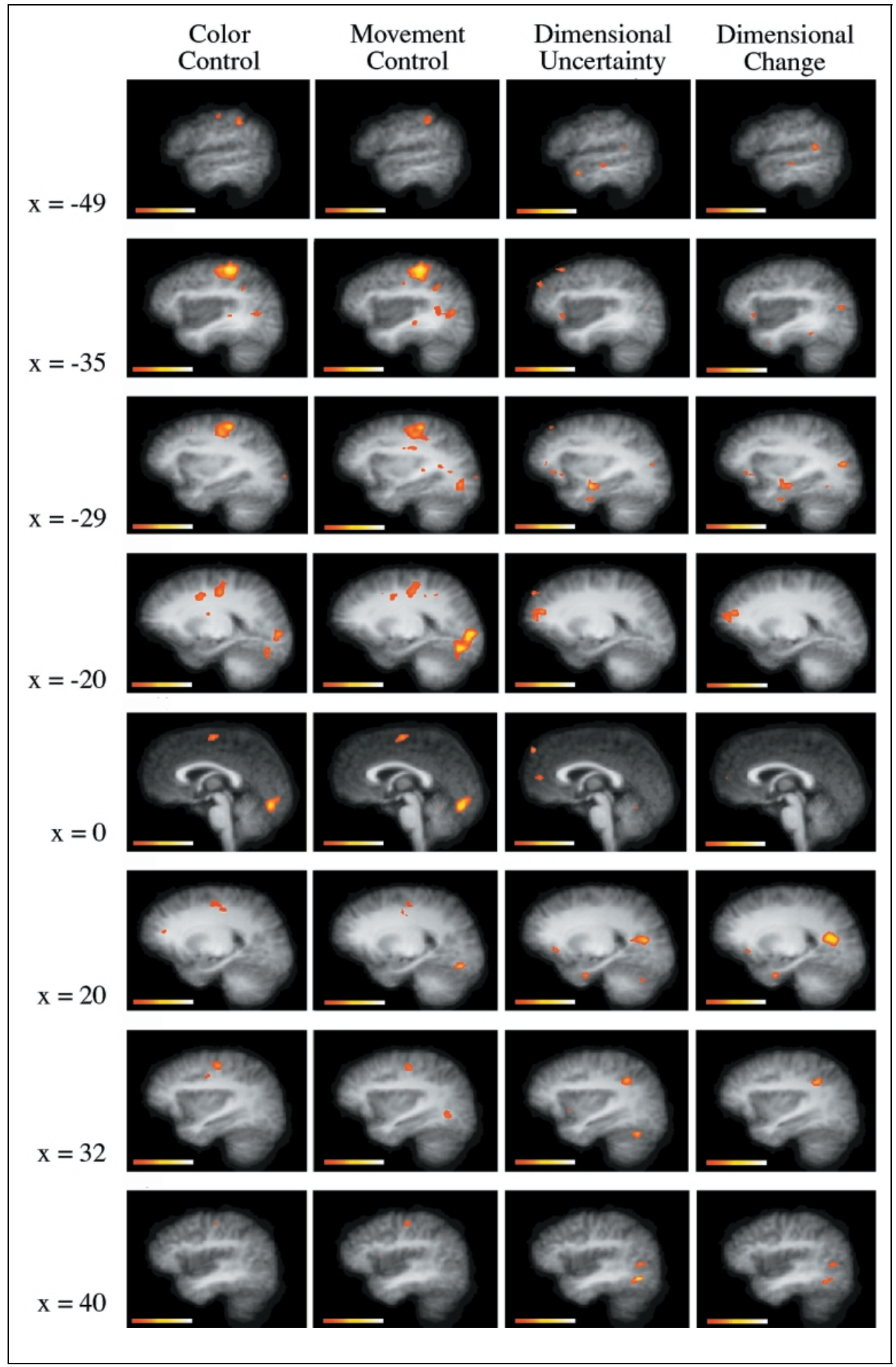


Table 1. Signal Increases in the Color Control Condition versus Fixation

\begin{tabular}{lcc}
\hline \multicolumn{1}{c}{ Structure } & Group z max & Location \\
\hline Cingulate sulcus, BA 10/32 & 5.44 & $\mathrm{R}(204011)$ \\
Anterior cingulate cortex, BA 24/32 & 6.17 & $\mathrm{R}(10371)$ \\
Middle frontal gyrus, BA 8/9 & 5.31 & $\mathrm{~L}(-252139)$ \\
Superior frontal gyrus, BA 6 & 10.66 & $\mathrm{~L}(-9-154)$ \\
Precentral gyrus, BA 6 & 5.76 & $\mathrm{R}(35-337)$ \\
Precentral gyrus, BA 4 & 9.30 & $\mathrm{~L}(-38-2354)$ \\
& 6.70 & $\mathrm{R}(33-1249)$ \\
Superior temporal sulcus, horizontal ramus, BA 19 & 5.34 & $\mathrm{R}(13-2456)$ \\
Lingual/fusiform gyrus/striate cortex BA 17/18 & 5.72 & $\mathrm{~L}(-37-6214)$ \\
Thalamus & 7.80 & $\mathrm{R}(2-80-2)$ \\
\hline
\end{tabular}

For each anatomical location, the maximal group $z$ - score is given, along with its location in the coordinate space of Talairach and Tournoux (1988). $\mathrm{L}=$ left-hemispheric; $\mathrm{R}=$ right-hemispheric activation. Only activations with a volume of $\geq 100 \mathrm{~mm}^{3}$ are presented.

(1996) and Müller et al. (1995) under fMRI scanner conditions.

\section{Functional-Imaging Data}

Activations Related to the Control Conditions with Fixed Target-Defining Dimension

Compared against the fixation baseline, a common network of activations was found when color or movement was the fixed target-defining dimension (Figure 3, Table 1, and Table 2). This network consisted bilaterally of the supplementary motor area (SMA)/ preSMA complex, the frontal eye fields, and lateral premotor cortex and, in the left hemisphere (opposite to the responding hand), motor cortex. Common activation was further evident in anterior cingulate cortex, in the horizontal ramus of the superior-temporal sulcus, and a widespread activation in ventral occipital cortex reaching up into striate cortex. This network reflects the demands on visual processing, motor preparation, and response execution common to both search tasks.

Table 2. Signal Increases in the Movement Control Condition versus Fixation

\begin{tabular}{lcc}
\hline \multicolumn{1}{c}{ Structure } & Group $z$ max & Location \\
\hline Anterior cingulate cortex, BA 24/32 & 5.76 & $\mathrm{R}(9350)$ \\
& 6.32 & $\mathrm{R}(142631)$ \\
Superior frontal gyrus, BA 6 & 11.00 & $\mathrm{~L}(-9-155)$ \\
Precentral gyrus, BA 4 & 8.95 & $\mathrm{~L}(-34-2355)$ \\
& 6.67 & $\mathrm{R}(36-1250)$ \\
Superior parietal lobule, BA 7/ & 6.93 & $\mathrm{~L}(-34-4339)$ \\
Intraparietal sulcus & 5.69 & $\mathrm{~L}(-23-4548)$ \\
& 6.58 & $\mathrm{R}(25-4838)$ \\
Superior temporal sulcus, horizontal ramus, BA 19 & 6.13 & $\mathrm{~L}(-44-452)$ \\
Cuneus, BA 18 & 5.57 & $\mathrm{R}(8-8420)$ \\
Lateral occipital sulcus, BA 37 & 6.17 & $\mathrm{R}(30-623)$ \\
Lingual/fusiform gyrus/striate cortex, BA 17/18 & 9.52 & $\mathrm{~L}(-11-84-1)$ \\
Parahippocampal gyrus, BA 27 & 5.47 & $\mathrm{~L}(-37-27-2)$ \\
\hline
\end{tabular}

For each anatomical location, the maximal group $z$ - score is given, along with its location in the coordinate space of Talairach and Tournoux (1988). $\mathrm{L}=$ left-hemispheric; $\mathrm{R}=$ right-hemispheric activation. Only activations with a volume of $\geq 100 \mathrm{~mm}^{3}$ are presented. 
Activations in the superior-parietal lobule, cuneus, and lateral occipital sulcus were observed only with search for movement-defined targets. The latter activation was just slightly anterior to previous reports of area V5 (Watson et al., 1993; Hasnain, Fox, \& Woldorff, 1998). Thus, parietal and dorsal-occipital cortices were preferentially activated when attention was directed to the movement dimension, consistent with previous reports (Büchel et al., 1998; Culham et al, 1998). Activations in the left middle frontal gyrus (BA 8/9) and left thalamus were selectively found with search for color-defined targets.

\section{Activations Related to Dimensional Change}

Brain areas involved in dimension shifting were expected to be active whenever the target on the current trial, $n$, was defined in a different dimension to that of the target on the immediately preceding trial, $n-1$ (e.g., motion-defined target on trial $n$, color-defined target on trial $n-1)$. To image these areas, an event-related analysis examined activation changes that occurred following target dimension changes (from movement to color and vice versa) relative to trial sequences without dimension changes (i.e., consecutive trials with repeated motion-defined or color-defined targets).

A large activation following dimensional change trials was observed in the left frontopolar cortex, at the junction of the superior frontal sulcus and the superior transverse frontopolar gyrus (Table 3, Figure 3). In this area, no signal changes were observed in the comparisons of the color and movement control conditions against fixation. Specifically, there was no evidence of deactivations, which have been reported to be common in this area (Shulman et al., 1997). Thus, the large dimensional change-related activation in the frontopolar cortex is not an indirect effect of deactivations elicited by search in the color or movement dimension. Other frontal activations were observed in the frontomedian wall at the anterior border of the cingulate cortex and bilaterally in inferior frontal gyrus.

Increased activation related to dimension changes was also found in the right superior parietal lobule and the intraparietal sulcus, as well as at the right hemispheric cuneus/precuneus border, at the bottom of the parietooccipital fissure and bilaterally along the horizontal ramus of the superior-temporal sulcus. Ventral to the latter activation, a change-related increase of activity was observed in the right hemisphere, the location of which $(x=39, y=-57, z=-3)$ coincides with the anterior border of a recently published variability map of the motion-sensitive area V5 (Hasnain et al., 1998). However, in the absence of independent functional-mapping experiments with our participants, this activation cannot be assigned to V5 unequivocally.

Increased activation following target dimension changes was revealed in several areas that are involved

Table 3. Event-Related Signal Increases Following Changes of the Target-Defining Dimension

\begin{tabular}{|c|c|c|}
\hline Structure & Group $z$ max & Location \\
\hline Frontopolar cortex, BA 10 & 6.86 & $\mathrm{~L}(-18506)$ \\
\hline Inferior frontal gyrus, BA 47 & 5.88 & $\mathrm{~L}\left(\begin{array}{lll}-39 & 19 & 0\end{array}\right)$ \\
\hline Inferior frontal gyrus, BA 11 & 5.91 & $R(2024-5)$ \\
\hline Anterior cingulate cortex, BA 32 & 5.34 & R (3 496$)$ \\
\hline Superior parietal lobule, BA 7 & 6.48 & $\mathrm{R}(9-4955)$ \\
\hline Superior parietal lobule, BA 7/intraparietal sulcus & 7.15 & $\mathrm{R}(32-4237)$ \\
\hline Precuneus, BA 31/cuneus, BA 18 & 7.97 & $\mathrm{R}(21-6121)$ \\
\hline Supramarginal gyrus, BA 42 & 6.61 & $\mathrm{~L}(-50-4419)$ \\
\hline Middle temporal gyrus, BA 21/inferior temporal sulcus & 5.85 & $L(-41-3-27)$ \\
\hline Superior temporal sulcus, & 7.87 & $\mathrm{~L}(-34-7023)$ \\
\hline horizontal ramus, BA 19 & 6.86 & $\mathrm{R}\left(\begin{array}{lll}41-60 & 17\end{array}\right)$ \\
\hline Lateral occipital sulcus, BA 37 & 5.98 & $\mathrm{R}(39-57-3)$ \\
\hline Fusiform gyrus, BA 37 & 5.44 & $\mathrm{~L}(-42-46-9)$ \\
\hline Hippocampus & 6.39 & $\mathrm{~L}(-30-20-9)$ \\
\hline \multirow[t]{2}{*}{ Parahippocampal gyrus, BA 35/36 } & 6.04 & $\mathrm{~L}(-31-13-24)$ \\
\hline & 6.51 & $\mathrm{R}(19-8-26)$ \\
\hline
\end{tabular}

For each anatomical location, the maximal group z- score is given, along with its location in the coordinate space of Talairach and Tournoux (1988). $\mathrm{L}=$ left-hemispheric; $\mathrm{R}=$ right-hemispheric activation. Only activations with a volume of $\geq 100 \mathrm{~mm}^{3}$ are presented. 
in the processing of motion and constitute part of the occipito-parietal ("where") pathway (Tootell et al., 1995; Ungerleider, Courtney, \& Haxby, 1998). Subsequent analyses examined whether these areas were selectively active during changes from color to motion (or, respectively, changes from motion to color). These separate analyses yielded no significant activations, probably due to lack of statistical power. However, the significant activation in the combined analysis of colorto-motion and motion-to-color changes, together with the absence of a signal increase in the analysis of colorto-motion changes alone, suggests that changes from both color to motion and motion to color elicited increased activation in these areas.

In the left hemisphere, increased activation was observed in middle-temporal gyrus, and in supramarginal gyrus. No activation changes related to target dimension changes were evident in the ventral occipital cortex, in or near the location of the visual color areas V4v/V8.

Dimensional change-related activation was also found bilaterally in the medial temporal lobes. Signal maxima were found in the left hippocampus and bilateral parahippocampal gyri. However, the loss of precision due to normalization and averaging prohibits an unequivocal localization in hippocampus proper.

In contrast to the increased activation in higher-level visual areas, there was a signal decrease related to target dimension changes in the striate cortex $(x=-2, y=$ $-85, z=6)$.

\section{Effects of Dimensional Uncertainty}

In addition to the event-related analysis of target dimension changes within the cross-dimensional search con-

Table 4. Signal Increases due to Dimensional Uncertainty

\begin{tabular}{|c|c|c|}
\hline Structure & Group $z \max$ & Location \\
\hline Superior frontal gyrus, BA 9 & 7.24 & $\mathrm{~L}(-185731)$ \\
\hline Superior frontal gyrus, BA 9/10 & 6.80 & $\mathrm{~L}\left(\begin{array}{lll}-15 & 46 & 19\end{array}\right)$ \\
\hline Medial frontal gyrus, BA 9 & 6.45 & $\mathrm{~L}(-15734)$ \\
\hline \multirow[t]{2}{*}{ Middle frontal gyrus, BA 9} & 6.29 & $\mathrm{~L}(-244335)$ \\
\hline & 6.42 & $\mathrm{~L}(-372944)$ \\
\hline Middle frontal gyrus, BA 10 & 6.29 & $\mathrm{~L}(-364926)$ \\
\hline Middle frontal gyrus, BA 10/46 & 6.70 & $\mathrm{~L}(-26425)$ \\
\hline \multirow[t]{2}{*}{ Inferior frontal gyrus, BA 11} & 6.07 & $\mathrm{~L}(-3027-2)$ \\
\hline & 6.48 & R (19 27-4) \\
\hline Anterior cingulate cortex, BA 32 & 5.95 & $\mathrm{R}(0445)$ \\
\hline Anterior cingulate cortex, BA 31 & 5.79 & R (15 18 28) \\
\hline Superior parietal lobule, BA 7 & 6.29 & $\mathrm{R}(10-5052)$ \\
\hline Superior parietal lobule, BA 7/intraparietal sulcus & 7.12 & $\mathrm{R}(33-4537)$ \\
\hline Precuneus, BA 31 & 7.08 & $\mathrm{R}(21-6620)$ \\
\hline Superior temporal sulcus, & 6.77 & $\mathrm{~L}(-34-6923)$ \\
\hline horizontal ramus, BA 19 & 6.77 & $\mathrm{R}(39-6214)$ \\
\hline \multirow[t]{2}{*}{ Superior temporal sulcus, BA 22} & 6.58 & $\mathrm{~L}(-481-15)$ \\
\hline & 5.47 & $\mathrm{~L}(-47-22-2)$ \\
\hline Lateral occipital sulcus, BA 37 & 8.44 & $\mathrm{R}(39-630)$ \\
\hline Fusiform gyrus, BA 36 & 6.55 & $\mathrm{~L}(-33-13-25)$ \\
\hline Putamen & 6.07 & $R(1512-6)$ \\
\hline Hippocampus & 7.40 & $\mathrm{~L}(-29-13-11)$ \\
\hline Parahippocampal gyrus, BA 28 & 6.48 & $\mathrm{R}(22-10-27)$ \\
\hline Cerebellum & 7.15 & $R(33-60-15)$ \\
\hline
\end{tabular}

For each anatomical location, the maximal group $\mathrm{z}$ - score is given, along with its location in the coordinate space of Talairach and Tournoux (1988). $\mathrm{L}=$ left-hemispheric; $\mathrm{R}=$ right-hemispheric activation. Only activations with a volume of $\geq 100 \mathrm{~mm}^{3}$ are presented. 
dition, we also compared the cross-dimensional condition as a whole to the joint color and movement control conditions, in which the target was defined by a fixed feature. This comparison yielded largely the same pattern of activation as the event-related analysis, including some additional activations not related to dimensional change (Table 4). Some overlap of the activations revealed in the dimensional-change and dimensionaluncertainty analyses was, of course, expected. Target dimension changes occurred only in the dimensionaluncertainty condition, so that change-related signal increases entailed signal increases in the uncertainty condition as a whole. However, dimensional uncertainty may itself lead to activation increases not specifically tied to dimensional change.

Such an increase was observed in an area in the frontomedian wall, at the anterior border of the left cingulate cortex (BA 24/32), which was strongly activated in the blockwise analysis, in accordance with previous findings of anterior cingulate activity during divided attention (e.g., Corbetta et al., 1991). By contrast, the extent of this activation was much smaller in the eventrelated analysis of target dimension changes (Figure 3). This is also illustrated by the almost flat time-course of
Figure 4. Selected BOLD-response timecourses exhibited by one participant. The panels show the unfiltered event-related BOLD-response (signal change in \%) as a function of time post stimulus onset (in msec) for dimensional changerelated activations in frontopolar, anterior cingulate, superior parietal and striate (deactivation) cortices. Slice-specific acquisition times account for the shifts in image acquisition time points between regions of interest.
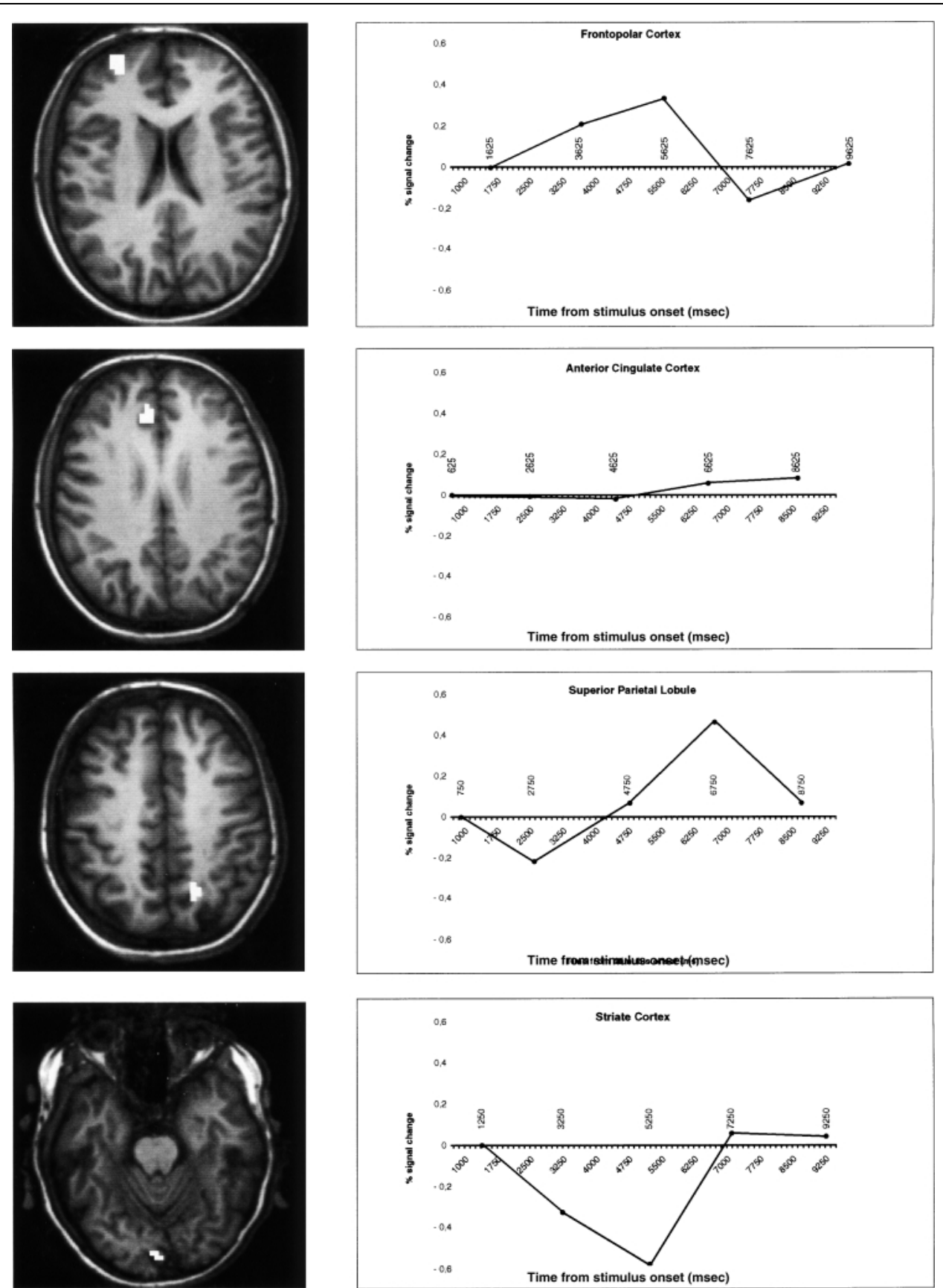

Pollmann et al. 
Figure 5. Tonic activation changes due to dimensional attention weighting were analyzed by comparing the color control and within color conditions to the movement control and within movement conditions. Positive activation values indicate higher activation in the joint color conditions, negative values indicate higher activation in the joint movement conditions. Since we specifically tested for differential activation in the regions of V4 and V5, the significance criterion was relaxed to $\alpha=$ .005 . (a) Attentive processing of color (compared to movement) led to increased activation in the fusiform gyri $(\mathrm{x}=-23$, $y=-64, z=-7 ; x=22$, $y=-76, z=-16$ ). (b) Attentive processing of movement (compared to color) was accompanied by increased activation along the right lateral occipital sulcus ( $x=41$, $y=-52, z=-2)$. The remaining activations lay outside the ROI. Left hemisphere is presented on the left.

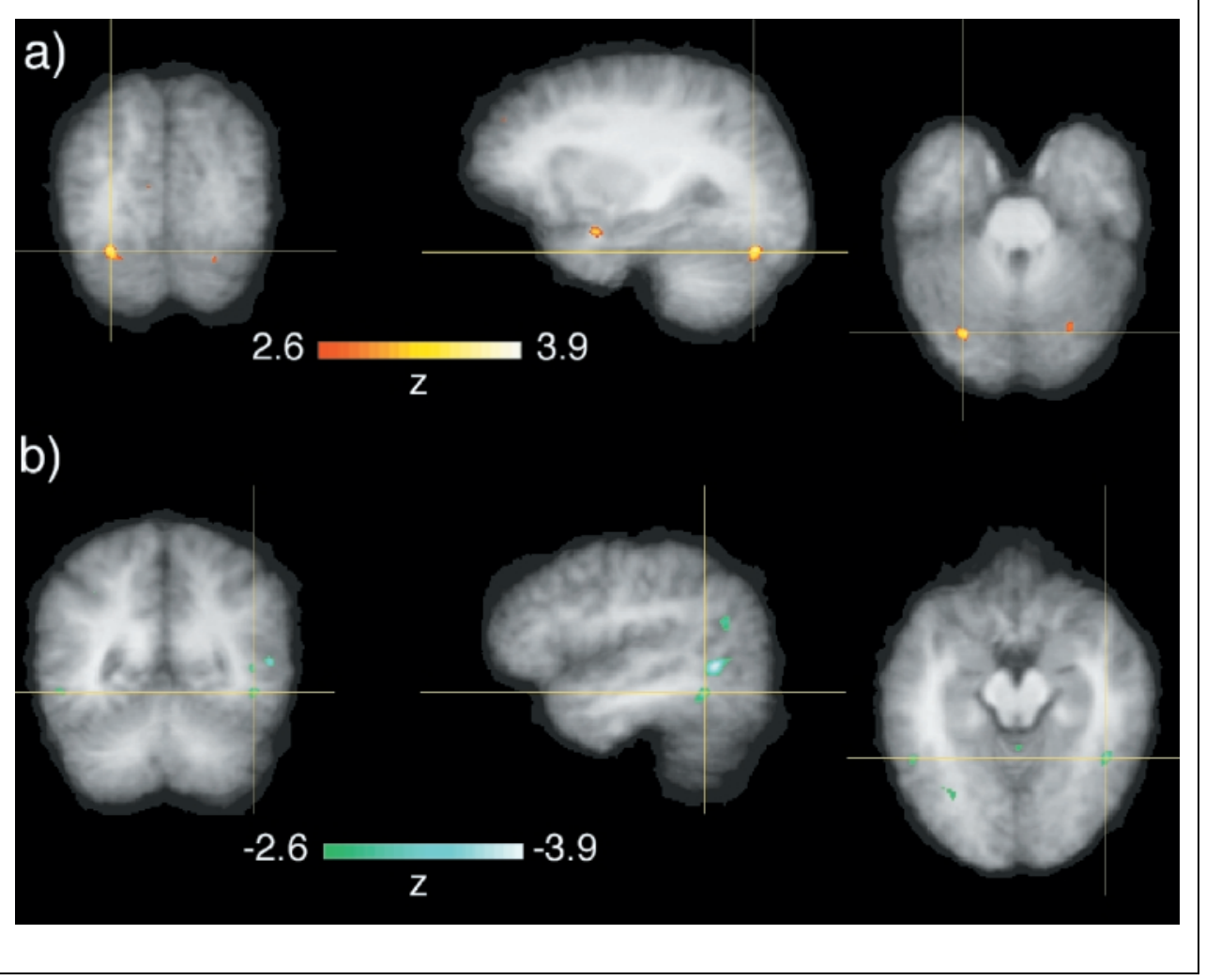

the BOLD-response exhibited by one participant in the activated area of the anterior cingulate (Figure 4). Spots of activation were also observed in left anterior superiorfrontal cortex (BA 9/10) and in left premotor cortex (BA 8). An additional activation was found in the anterior portion of the left middle-temporal gyrus.

\section{Effects of Featural Uncertainty}

With featural uncertainty within a constant dimension (variable red or blue color targets, or variable lowerleft-to-upper-right or upper-left-to-lower right movement targets), the pattern of activations did not differ from that in the respective color and movement control conditions. Further, an event-related analysis carried out for the within-dimension conditions yielded no activations on feature change trials relative to no-change trials, in contrast to the analysis for the cross-dimension conditions. These data are consistent with the lack of featural uncertainty effects in the RT data. Taken together, this pattern of results confirms that the activation changes outlined above are specific to cross-dimensional shifts.

\section{Attentional Weighting of Target Dimensions}

According to the dimension weighting account, changes of the target-defining dimension involve the shifting of attentional weight to the new target dimension, accom- panied by increased activation in brain areas dedicated to processing the new dimension, for as long as this dimension remains unchanged. We analyzed such tonic attention effects by contrasting the color and movement control conditions in a region-of-interest analysis. Regions of interest were the human color-sensitive area V4 and the movement-sensitive area V5.

Higher activation for search in the color dimension, compared to search in the movement dimension, at or near previously reported locations of V4 (Hasnain et al., 1998; McKeefry \& Zeki, 1997) were observed in left fusiform gyrus at $x=-23, y=-64, z=-7$ and in right fusiform gyrus at $x=22, y=-76, z=-16$ (Figure 5).

Near previously reported locations of V5 (Hasnain et al., 1998; Watson et al., 1993), search in the movement dimension (compared to the color dimension) elicited significantly higher activation along the right lateral occipital sulcus at $x=41, y=-52, z=-2$.

\section{DISCUSSION}

\section{Frontal Cortex Involvement in Cross-Dimensional Search}

Target dimension changes were accompanied by activation in a network of brain structures, ranging from the frontal to occipital cortex. In the frontal cortex, mainly two structures showed increased activation when the target-defining dimension was variable (either color or movement), compared with when it was fixed. One 
activation was found in the left frontopolar cortex (BA 10), the other in the frontomedian wall at the anterior border of the anterior cingulate cortex (BA 24/32). These two structures seem to play complementary roles in the allocation of attentional resources when target stimuli may appear in either of two dimensions.

While the anterior cingulate cortex was activated under cross-dimensional search conditions compared with within-dimensional search, it showed only a weak increase of activation following actual dimension changes. In other words, the activation was increased throughout the cross-dimensional search block, independent of whether a dimensional change occurred or not. In contrast, frontopolar activation was increased when the target dimension changed, compared with when the target dimension remained unchanged.

This suggests that anterior cingulate cortex activity represents a continuous "divided-attention" set (Corbetta et al., 1991) that enables attentional weight to be shifted to the relevant feature dimensions, whereas the frontopolar cortex is involved during the actual shifting of attentional weight.

A further activation related to dimensional change was found in the inferior frontal gyri, an area that has previously been reported to be active under cross-dimension search conditions (Corbetta et al., 1991). The present analyses revealed this activation to be related to actual dimensional change, rather than dimensional uncertainty as such. In terms of the distinction developed above, this would mean that the inferior frontal gyri are also involved in the process of redirecting attentional weight to from the old to the new dimension.

Recently, several foci of activations extending from the orbitofrontal cortex into the middle-frontal gyrus (BA 10) have been reported in a spatial cueing study, when invalid spatial cues misdirected attention away from the target location (Nobre, Coull, Frith, \& Mesulam, 1999). Common to this study and ours was an activation in BA 10 in the left hemisphere. However, the precise locations of activation differed in that invalid spatial cues led to activations in orbitofrontal cortex and middle-frontal gyrus, whereas visual dimension changes led to frontopolar activation. This anatomical difference may be related to a difference in the nature of the attentional processes required by the tasks in the two studies: Reallocation of attention from a cued location to an uncued location (i.e., space-based selection) in the study of Nobre et al., in contrast with reallocation of attention from a visual dimension that defined the target on the preceding trial to a new target-defining dimension (i.e., dimension-based selection) in the present study.

Nobre et al. (1999) discussed "breaches of expectation" as a possible reason for their orbitofrontal activation. While the foveally presented cues in their experiment predicted the target location with a high validity, in our cross-dimension condition the target on a given trial was equally likely to be defined in either of the two dimensions, irrespective of the target dimension on the preceding trial. That is, our participants (who were informed of this contingency) had no reason to expect a repetition of the target-defining dimension. Furthermore, assuming that our participants had nevertheless expected a target repetition, they should have done so not only under cross-dimension (dimension repetition), but also under within-dimension search conditions (feature repetition). However, within-dimension search revealed no frontopolar activation following feature changes across consecutive trials, despite the feature changes being matched in frequency and sequencing with cross-dimensional changes. Finally, in singleton feature search experiments, participants do not explicitly encode and retain the target dimension on a given trial (Müller et al., 1999). Thus, any dimensional "expectations" would be implicit in nature, realized in terms of the attentional weighting of the current dimension. We therefore favor the view that the frontopolar cortex is active only when attention has to be reallocated. Thus, while it cannot be ruled out that "breaches of [spatial] expectation" lead to activation in the orbitofrontal cortex, the frontopolar cortex is involved in the reallocation of attentional resources between visual dimensions.

Nobre et al. (1999) also suggested that the orbitofrontal cortex may be needed to redirect or withhold responses. In our study, the target-defining dimension was entirely irrelevant for the required response-participants had simply to detect and make the same keypress response to any odd-one-out target whatever its defining dimension. There is a parallel in the spatial cueing experiment of Nobre et al., in that target detection required the same keypress response whatever the target location. Thus, in both studies, the frontopolar and, respectively, orbitofrontal cortex were active in situations requiring reallocation of attention, but not inhibition or redirection of responses.

\section{Involvement of Posterior Visual Areas in Dimensional Change}

Dorsal occipital visual areas exhibited increased activation during target dimension changes. Since these areas contain motion-selective neurons, they might have been expected to be selectively active during color-to-motion changes, when attentional weight is reallocated to the movement dimension. However, increased activation in these areas was also observed during changes from motion to color. Moreover, dorsal occipital activation was complemented by posterior parietal activation, both at the banks of the intraparietal sulcus (at the border of the horizontal to ascending portion), in the precuneus, and at the cuneus/precuneus border, during target dimension changes. While there is good evidence for a role of the posterior parietal cortex in shifts of spatial attention (Corbetta, Miezin, Shulman, \& Petersen, 1993; Cor- 
betta et al., 1998; Petersen, Corbetta, Miezin, \& Shulman, 1994), it has also been shown to be involved in nonspatial attention (Coull \& Frith 1998), particularly nonspatial attention shifts (Dove, Pollmann, Schubert, Wiggins, \& von Cramon, 2000; Le, Pardo, \& Hu, 1998). The present data extend these findings by showing that the posterior parietal cortex, together with occipital areas belonging to the dorsal ("where") pathway, are involved in nonspatial shifts of attentional weight between feature dimensions.

In summary, target dimension changes led to increased activation in higher-level temporal and parietal processing areas, that is, both in the ventral and dorsal pathways. While increased change-related activation was observed in or near the motion-responsive area V5, this was not the case in ventral occipital cortex, in or near the color-processing areas (V4v/V8). Attentional modulation has previously been observed both in V5 (Beauchamp, Cox, \& De Yoe, 1997; O'Craven, Rosen, Kwong, Treisman, \& Savoy, 1997) and V4 ( Martinez et al., 1999; Kastner, de Weerd, Desimone, \& Ungerleider, 1998), ruling out a general lack of attentional modulation of V4 as a cause for this asymmetry.

The striate cortex exhibited reduced activation during changes of the target dimension. Striate deactivation has been observed following eye movements (Paus, Marrett, Worsley, \& Evans, 1995). It is unlikely, however that eye movements were more frequent following dimension changes in the current task. Eye movements reliably activate the frontal eye fields (FEF), at the junction of the precentral and superior-frontal sulci (Luna et al., 1998; Paus, 1996), and we have reliably observed FEF activation in previous fMRI studies of visual search for conjunction targets, requiring overt saccades (Pollmann \& von Cramon, in press; Pollmann, Wiggins, Norris, von Cramon, \& Schubert, 1998). FEF activity was also manifest in the present study, for both the color and the movement control condition relative to fixation. However, FEF activation was not found to be associated with target dimension changes, indicating that dimensional change did not lead to increased oculomotor activity. Thus, rather than being due to an increase in eye movements, the change-related deactivation in striate cortex may be indicative of suppression of the irrelevant dimension.

ERP studies have provided evidence for attentional selection involving both enhancement of relevant attributes of the attended stimulus and suppression of irrelevant attributes, with suppression preceding facilitation (Hillyard \& Anllo-Vento, 1998). Recently, a combined ERP and fMRI study has provided evidence that attentional facilitation occurs in extrastriate visual areas at the earliest, presumably driven by re-entrant feedback from higher visual areas (Martinez et al., 1999). It has been suggested that suppression effects should be prevalent in lower-tier visual areas, which are activated to near maximum by physical stimulus presentation alone (especially with physically intense stimuli), leaving more room for attentional suppression of activity than for enhancement (Posner \& Dehaene, 1994). Although attentional selection by location (Gandhi et al., 1999; Martinez et al., 1999, Somers et al., 1999) and features (Jäncke et al., 1999; Watanabe et al., 1998) leads to increased activation in striate cortex, the increases were small, especially when compared with higher-tier visual processing areas (Büchel et al., 1998). A recent study of spatial-attentional selection demonstrated specific facilitatory and inhibitory effects of spatial attention throughout the retinotopic visual areas. When attention was directed to a quadrant of the visual field, retinotopic attention effects were observed within retinotopic visual cortical areas: BOLD-responses were increased in areas representing the attended quadrant, but decreased in the other areas representing the unattended quadrants. However, while the signal increase in the attended quadrant was rather weak in V1, and increased gradually in V2, V3/Vp, V3a/V7, and V4v/V8 (Tootell et al., 1998, p. 1416), signal reduction in the unattended quadrants was relatively small and approximately equal in all retinotopic areas. V1 was the only area with larger decreases than increases. A deactivation in V1 has also been reported during spontaneous reversals of the perception of ambiguous figures (Kleinschmidt, Büchel, Zeki, \& Frackowiak, 1998). Thus, switching between visual dimensions of a stimulus, or switching between different percepts of a stimulus, may both lead to short-term suppression in V1.

\section{Medial-Temporal Lobe Activation Following Di- mensional Change}

Dimensional change led to bilateral phasic BOLD increases in the medial temporal lobes. The human hippocampal region has been shown to play an essential role in the detection and response to novel stimuli. It is engaged in the orienting response to perturbations in the stream of visual events (Knight, 1996). Although the medial temporal lobes become engaged automatically whenever an event is experienced, the strength of activation depends on familiarity: Novel events elicit greater activation than familiar ones (Martin, 1999). This is consistent with the phasic activation of the medial temporal lobes following changes to a new target-defining dimension.

\section{Attentional Weighting of a Visual Dimension}

When movement was the fixed target dimension, compared to when color was the target dimension, significantly increased activation was observed in the right lateral occipital sulcus. The location of this activation was at the anterior border of a previously reported variability map of human V5 (Hasnain et al., 1998). When color was the constant target dimension, relative to when movement was the target dimension, increased 
activation was evident bilaterally in the fusiform gyri at locations corresponding to previously reported coordinates of the color sensitive area V4. Without independent functional mapping of V4 and V5 in our participants, it remains questionable whether these activations are actually within these functional areas, or in their close neighborhoods. Nevertheless, it is clear that attentive processing of the color dimension led to increased activation in ventral occipital cortex, whereas attentive processing of the movement dimension led to increased activation in dorsal occipital cortex. This was predicted by the attentional dimension-weighting account.

\section{Summary}

Changes in the dimension defining singleton feature targets led to increased activation in a fronto-posterior network consisting of left frontopolar cortex and inferior-frontal gyri, high-level visual processing areas in parietal and temporal cortex, and dorsal occipital visual areas. When attentional weight was shifted to a new target-defining dimension, activation increased in the visual areas involved in processing features of this dimension. In addition, activation in the primary visual cortex was transiently reduced.

We hypothesize that frontopolar cortex is involved in controlling attentional weight shifting and that inferior frontal gyri and high-level parietal and temporal areas mediate attentional weighting via feedback to extrastriate visual areas that process the features of the new target dimension. Reduced activation in the striate cortex may indicate attentional selection by suppression of features of the old dimension.

\section{METHODS}

Ten participants took part in the fMRI experiment, each having given prior informed consent according to the Max-Planck-Institute guidelines. Six of the participants were female and four male. Participants' ages ranged between 18 and 27 years, with a mean age of 23.6 years. All participants were right-handed, assessed by means of the Edinburgh Inventory (Oldfield, 1971).

Stimuli were displayed by an LCD projector on a back-projection screen mounted in the bore of the magnet behind the participants' head. Participants viewed the screen wearing mirror glasses, which were equipped with corrective lenses if necessary. Each trial began with the presentation of a display matrix of $6 \times 6$ squares, covering an area of $7.7 \times 7.7^{\circ}$ (Figure 1). All distractor squares were green and moved sinusoidally along their horizontal axis (maximum amplitude $=0.4^{\circ}$, speed $=1 \%$ ). In the color control condition, the target square was red (while moving horizontally). In the movement control condition, the target moved sinusoidally along the lower-left upper-right diagonal axis (while being colored green). Feature uncertainty was realized in two within-dimension conditions. In the within-color condition, the target could be either red or blue. In the within-movement condition, the target moved sinusoidally along the lower-left upper-right or the upper-left lower-right diagonal axis. In the critical cross-dimension search condition, the target-defining dimension varied randomly across trials, that is, the target was either colored red or moved along the lower-left upper-right diagonal axis. The colors were equiluminant (red: 21.0 $\mathrm{cd} / \mathrm{m}^{2}$, CIE coordinates $x=0.583$ and $y=0.329$; green: $21.2 \mathrm{~cd} / \mathrm{m}^{2}, x=0.248, y=0.587$; blue: $21.3 \mathrm{~cd} / \mathrm{m}^{2}, x=$ $0.143, y=0.074)$. The stimulus display was terminated by the participant's response or after a maximum of 1.5 sec, and replaced by a central fixation mark that stayed on until the start of the next trial. Trial duration was always 2 sec.

A session consisted of six scans, each scan starting with $30 \mathrm{sec}$ of fixation followed by 10 task blocks. Each block consisted of 20 trials. Blocks were separated by 10 sec of fixation. Before each block, the experimental condition was indicated to the participant by a letter cue presented for $10 \mathrm{sec}$. The order of experimental conditions was varied in the following way. In one half of the scans, participants started with the control condition (a color control block and a movement control block) followed by the within condition (two within-color blocks and two within-movement blocks) and the cross-dimension condition (four blocks). In the other half, the order of conditions was reversed. Presentation order alternated in consecutive scans. In the withindimension conditions, the target-defining feature could change from trial to trial (90 feature changes per participant in the within dimension blocks). In the crossdimension condition, the target-defining dimension could change (90 dimension changes per participant in the cross-dimension blocks).

Participants were instructed to give a speeded forcedchoice response using the index or middle finger of their right hand to indicate the presence or, respectively, absence of a singleton feature target. Which specific dimension or feature defined the target was irrelevant for the target-present response. One day before the fMRI experiment, participants took part in a training session, which consisted of the same condition and trial sequence that was subsequently presented in the scanner.

Sixteen axial slices were acquired parallel to the AC-PCplane. Slice thickness was $5 \mathrm{~mm}$, interslice distance $2 \mathrm{~mm}$, with a $19.2 \mathrm{~cm}$ FOV and an image matrix of $64 \times 64$. Data were collected at $3 \mathrm{~T}$ by a Bruker 30/100 Medspec system (Bruker Medizintechnik, Ettlingen, Germany), using a gradient-recalled EPI sequence $(\mathrm{TR}=2000 \mathrm{msec}, \mathrm{TE}=$ $40 \mathrm{msec}$, flip angle $=40^{\circ}$ ). Data were corrected for movement artefacts according to the algorithm of Friston, Williams, Howard, Frackowiak, \& Turner, 1996 and filtered with a spatial Gaussian filter with FWHM $=2.35 \mathrm{~mm}$. 


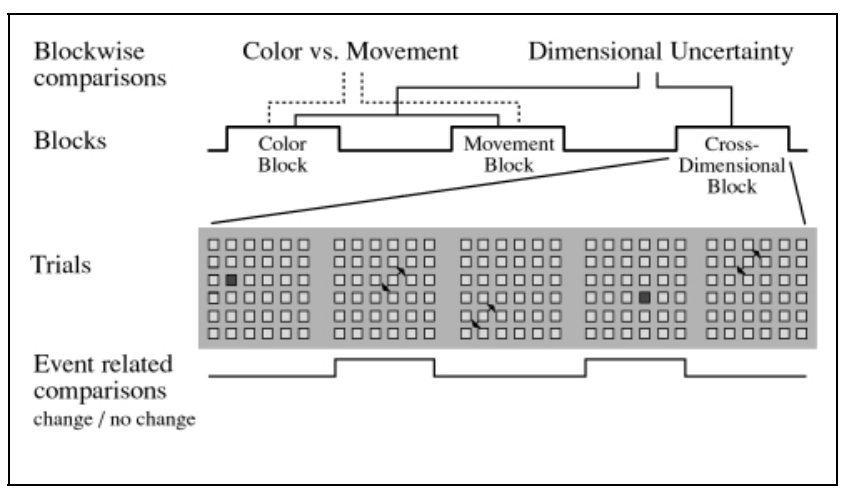

Figure 6. Illustration of experimental design and blockwise and eventrelated comparisons. Blockwise comparisons between experimental conditions examined effects of dimensional uncertainty and attentional weighting of the color and movement dimension, respectively, and event-related analyses examined phasic activation changes following changes of the target-defining feature or dimension, respectively. For simplicity, the color and movement control (fixed dimension, fixed feature) and within-dimension (fixed dimension, variable feature) blocks are not depicted separately. On average, a feature or dimension change occurred every 5.3 trials.

To assess activation changes associated with the control conditions, activations during the color and, respectively, movement control blocks were compared with their preceding fixation periods. Effects of featural uncertainty were examined by comparisons between the within-color and color control conditions and the within-movement and movement control conditions (Figure 6). Effects of dimensional uncertainty were examined by comparing the cross-dimensional search condition with the combined color and movement control conditions. In addition to the blockwise analyses, event-related analyses of target feature and dimension changes were carried out within the within-dimension and cross-dimensional uncertainty blocks. All trials in the withindimension conditions in which the target-defining feature was different from the target feature in the immediately preceding trial were marked as featural change trials. Likewise, all trials in the cross-dimensional search condition in which the target-defining dimension was different from the target dimension in the immediately preceding trial were marked as dimensional change trials. We analyzed changes in the BOLD-response, which were elicited by featural and dimensional change trials embedded in a rapid sequence of search trials without change in the target-defining feature or dimension. A correlation analysis was calculated between the time course of the acquired BOLD-response and an idealized time course. The latter was generated by specifying the images acquired during changes of target dimension as "on"-states and the remaining images in the cross-dimension search condition as "off"-states (Figure 6). This boxcar function was convolved with a Gaussian model of the hemodynamic response (lag $=4.8$ sec, $\sigma=1.8$ sec; cf. Clark, Maisog, \& Haxby, 1998).
Analogous analyses were carried out for featural changes in the featural, within-color and within-movement, uncertainty conditions. A previous study (Pollmann, Dove, von Cramon, \& Wiggins, 2000) had shown that overlap of successive BOLD-responses does not affect the comparison of signal differences between experimental conditions: neither amplitude differences nor peak latencies did change, whether stimuli were presented one every 16 sec, allowing the BOLD-response to return to baseline during the inter-stimulus interval, or in rapid succession, one every $2 \mathrm{sec}$, as in the present experiment.

Degrees of freedom were corrected for temporal autocorrelation of the BOLD-response (Worsley \& Friston, 1996). Average $z$ maps were computed, after registration with individual $3 \mathrm{D}$ anatomical images and normalization by linear scaling, by performing $t$ tests for known variance at corresponding voxels across participants. The resulting group $z$ value over $n$ participants equals the average individual $z$ value $* \sqrt{ } n$ (Bosch, 2000). To correct for multiple spatial comparisons, the significance criterion for whole brain analyses was $\alpha<.000001$. For region-of-interest analyses, the significance criterion was set to $\alpha=.005$. Region-ofinterest analyses were carried out to examine activation differences in V4 and V5 between the color and movement control conditions. The group $z$ maps were superimposed on the averaged normalized high-resolution $3 \mathrm{D}$ anatomical data sets of the participants.

\section{Acknowledgments}

This study was supported by a grant from the Deutsche Forschungsgemeinschaft (DFG Working Memory Research Group grant SCHR 375/8-1). We thank Glyn Humphreys and two anonymous reviewers for their helpful comments on a previous version of this manuscript.

Reprint requests should be sent to: Stefan Pollmann, MaxPlanck-Institute of Cognitive Neuroscience, Stephanstrasse 1a, D-04103 Leipzig, Germany; e-mail: pollmann@cns.mpg.de.

\section{REFERENCES}

Beauchamp, M. S., Cox, R. W., \& DeYoe, E. A. (1997). Graded effects of spatial and featural attention on human area MT and associated motion processing areas. Journal of Neurophysiology, 78, 516-520.

Bosch, V. (2000). Statistical analysis of multi-subject fMRI data: The assessment of focal activations. Journal of Magnetic Resonance, 11, 61-64.

Büchel, C., Josephs, O., Rees, G., Turner, R., Frith, C. D., \& Friston, K. J. (1998). The functional anatomy of attention to visual motion: A functional MRI study. Brain, 121, 12811294.

Clark, V. P., Maisog, J. M., \& Haxby, J. V. (1998). FMRI study of face perception and memory using random stimulus sequences. Journal of Neurophysiology, 79, 3257-3265.

Corbetta, M., Akbudak, E., Conturo, T. E., Snyder, A. Z., Ollinger, J. M., Drury, H. A., Linenweber, M. R., Petersen, S. E., Raichle, M. E., Van Essen, D. C., \& Shulman, G. L. (1998). A 
common network of functional areas for attention and eye movements. Neuron, 21, 761-773.

Corbetta, M., Miezin, F. M., Dobmeyer, S., Shulman, G. L., \& Petersen, S. (1991). Selective and divided attention during visual discriminations of shape, color, and speed: Functional anatomy by Positron Emission Tomography. Journal of Neuroscience, 11, 2383-2402.

Corbetta, M., Miezin, F. M., Shulman, G. L., \& Petersen, S. E. (1993). A PET-study of visuospatial attention. Journal of Neuroscience, 13, 1202-1226.

Coull, J. T., \& Frith, C. D. (1998). Differential activation of right superior parietal cortex and intraparietal sulcus by spatial and nonspatial attention. Neuroimage, 8, 176-187.

Culham, J. C., Brandt, S. A., Cavanagh, P., Kanwisher, N. G., Dale, A. M., \& Tootell, R. B. H. (1998). Cortical fMRI activation produced by attentive tracking of moving targets. Journal of Neurophysiology, 80, 2657-2670.

Dove, A., Pollmann, S., Schubert, T., Wiggins, C. J., \& von Cramon, D. Y. (2000). Prefrontal cortex activation in taskswitching. Cognitive Brain Research, 9, 103-109.

Duncan, J., \& Humphreys, G. W. (1989). Visual search and stimulus similarity. Psychological Review, 96, 433-458.

Found, A., \& Müller, H. J. (1996). Searching for unknown feature targets on more than one dimension: Investigating a "dimension-weighting" account. Perception and Psychophysics, 58, 88-101.

Friston, K. J., Williams, S., Howard, R., Frackowiak, R. S. J., \& Turner, R. (1996). Movement-related effects in fMRI timeseries. Magnetic Resonance in Medicine, 35, 346-355.

Gandhi, S., Heeger, D. J., \& Boynton, G.M. (1999). Spatial attention affects brain activity in human primary visual cortex. Proceedings of the National Academy of Sciences, U.S.A., 96, 3314-3319.

Grossberg, S., Mignolla, E., \& Ross, W. D. (1994). A neural theory of attentive visual search: Interaction of boundary, surface, spatial, and object representations. Psychological Review, 101, 470-498.

Hasnain, M. K., Fox, P. T., \& Woldorff, M. G. (1998). Intersubject variability in the human visual cortex. Human Brain Mapping, 6, 301-315.

Hillyard, S. A., \& Anllo-Vento, L. (1998). Event-related potentials in the study of visual selective attention. Proceedings of the National Academy of Sciences, U.S.A., 95, 781-787.

Jäncke, L., Mirzazade, S., \& Shah, N. J. (1999). Attention modulates the blood oxygen level dependent response in the primary visual cortex measured with functional magnetic resonance imaging. Naturwissenschaften, 86, 79-81.

Kastner, S., de Weerd, P., Desimone, R., \& Ungerleider, L. (1998). Mechanisms of directed attention in the human extrastriate cortex as revealed by functional MRI. Science, 282, 108-111.

Kleinschmidt, A., Büchel, C., Zeki, S., \& Frackowiak, R. S. J. (1998). Human brain activity during spontaneously reversing perception of ambiguous figures. Proceedings of the Royal Society, London, B, 265, 2427-2433.

Knight, R. (1996). Contribution of human hippocampal region to novelty detection. Nature, 383, 256-259.

Krummenacher, J., Müller, H. J., \& Heller, D. (submitted). Visual search for dimensionally redundant pop-out targets: Evidence for parallel-interactive processing of dimensions.

Le, T. H., Pardo, J. V., \& Hu, X. (1998). 4T-fMRI study of nonspatial shifting of selective attention: Cerebellar and parietal contributions. Journal of Neurophysiology, 79, 1535-1548.

Luna, B., Thulborn, K. R., Strojwas, M. H., McCurtain, B. J., Berman, R. A., Genovese, C. R., \& Sweeney, J. A. (1998). Dorsal cortical regions subserving visually guided saccades in humans: An fMRI study. Cerebral Cortex, 8, 40-47.

Martin, A. (1999). Automatic activation of the medial temporal lobe during encoding: Lateralized influences of meaning and novelty. Hippocampus, 9, 62-70.

Martinez, A., Anllo-Vento, L., Sereno, M. I., Frank, L. R., Buxton, R. B., Dubowitz, D. J., Wong, E. C., Hinrichs, H., Heinze, H. J., \& Hillyard, S. A. (1999). Involvement of striate and extrastriate visual cortical areas in spatial attention. Nature Neuroscience, 2, 364-369.

McKeefry, D. J., \& Zeki, S. (1997). The position and topography of the human colour centre as revealed by functional magnetic resonance imaging. Brain, 120, 2229-2242.

Müller, H. J., Heller, D., \& Ziegler, J. (1995). Visual search for singleton feature targets within and across feature dimensions. Perception and Psychopbysics, 57, 1-17.

Müller, H. J., Krummenacher, J., \& Heller, D. (submitted). Dimension-specific inter-trial facilitation in visual search for pop-out targets: Evidence for an implicit short-term memory effect.

Nobre, A. C., Coull, J. T., Frith, C. D., \& Mesulam, M. M. (1999). Orbitofrontal cortex is activated during breaches of expectation in tasks of visual attention. Nature Neuroscience, 2, 11-12.

O’Craven, K. M., Rosen, B. R., Kwong, K. K., Treisman, A., \& Savoy, R. L. (1997). Voluntary attention modulates fMRI activity in human MT-MST. Neuron, 18, 591-598.

Oldfield, R. C. (1971). The assessment and analysis of handedness: The Edinburgh Inventory. Neuropsychologia, 9, 97113.

Paus, T. (1996). Location and function of the human frontal eye field: A selective review. Neuropsychologia, 34, 475-483.

Paus, T., Marrett, S., Worsley, K. J., \& Evans, A. C. (1995). Extraretinal modulation of cerebral blood flow in the human visual cortex: Implications for saccadic suppression. Journal of Neurophysiology, 74, 2179-2183.

Petersen, S. E., Corbetta, M., Miezin, F. M., \& Shulman, G. L. (1994). PET studies of parietal involvement in spatial attention: Comparison of different task types. Canadian Journal of Experimental Psychology, 48, 319-38.

Pollmann, S., Dove, A., von Cramon, D. Y., \& Wiggins, C. J. (2000). Event-related fMRI: Comparison of conditions with varying BOLD-overlap. Human Brain Mapping, 9, 26-37.

Pollmann, S., Wiggins, C. J., Norris, D. G., von Cramon, D. Y., \& Schubert, T. (1998). Use of short intertrial intervals in singletrial experiments: A 3T fMRI-study. Neuroimage, 8, 327-339.

Pollmann, S., \& von Cramon, D. Y. (in press). Object working memory and visuospatial processing: Functional neuroanatomy analysed by event-related fMRI. Experimental Brain Research.

Posner, M. I., \& Dehaene, S. (1994). Attentional networks. Trends in Neurosciences, 17, 75-79.

Reimann, B., Müller, H. J., \& Krummenacher, J. (in preparation). Stimulus-driven and expectancy-driven effects in dimensional weighting.

Shulman, G. L., Fiez, J. A., Corbetta, M., Buckner, R. L., Miezin, F. M., Raichle, M. E., \& Petersen, S. E. (1997). Common blood flow changes across visual tasks: II. Decreases in cerebral cortex. Journal of Cognitive Neuroscience, 9, 648-663.

Somers, D. C., Dale, A. M., Seiffert, A. E., \& Tootell, R. B. H. (1999). Functional MRI reveals spatially specific attentional modulation in human primary visual cortex. Proceedings of the National Academy of Sciences, U.S.A., 96, 1663-1668.

Talairach, J., \& Tournoux, P. (1988) Co-planar stereotactic atlas of the buman brain. Stuttgart: Thieme.

Tootell, R. B. H., Hadjikhani, N., Hall, E. K., Marett, S., Vanduffel, W., Vaughan, J. T., \& Dale, A. (1998). The retinotopy of visual spatial attention. Neuron, 21, 1409-1422.

Tootell, R. B., Reppas, J. B., Kwong, K. K., Malach, R., Born, R. T., Brady, T. J., Rosen, B. R., \& Belliveau, J. W. (1995).

Functional analysis of human MT and related visual cortical 
areas using magnetic resonance imaging. Journal of $\mathrm{Neu}$ roscience, 15, 3215-3230.

Treisman, A. (1988). Features and Objects: The fourteenth Bartlett memorial lecture. Quarterly Journal of Experimental Psychology, 40A, 201-237.

Treisman, A. M., \& Gelade, G. (1980). A feature-integration theory of attention. Cognitive Psychology, 12, 97-136.

Ungerleider, L. G., Courtney, S. M., \& Haxby, J. V. (1998). A neural sytem for human visual working memory. Proceedings of the National Academy of Sciences, U.S.A., 95, 883-90.

Watanabe, T., Harner, A. M., Miyauchi, S., Sasaki, Y., Nielsen, M., Palomo, D., \& Mukai, I. (1998). Task-dependent influ- ences of attention on the activation of human primary visual cortex. Proceedings of the National Academy of Sciences, U.S.A., 95, 11489-11492.

Watson, J. D. G., Myers, R., Frackowiak, R. S. J., Hajnal, J. V., Woods, R. P., Mazziotta, J. C., Shipp, S., \& Zeki, S. (1993). Area V5 of the human brain: Evidence from a combined study using positron emission tomography and magnetic resonance imaging. Cerebral Cortex, 3, 79-94.

Wolfe, J. M. (1994). Guided search 2.0.: A revised model of visual search. Psychonomic Bulletin and Review, 1, 202238.

Worsley, K. J., \& Friston, K. J. (1995). Analysis of fMRI timeseries revisited-again. Neurolmage, 2, 173-181. 